\title{
EL INSTINTO DE TRABAJO ÚTIL Y EL FASTIDIO DEL TRABAJO*
}

\section{Thorstein Veblen}

Uno de los lugares comunes de la teoría económica recibida es que el trabajo es fastidioso. Muchos análisis parten del axioma de que, en relación con los asuntos económicos, los hombres desean sobre todas las cosas conseguir los bienes producidos por el trabajo y evitar el trabajo mediante el cual se producen esos bienes. En un sentido general, la opinión de sentido común se corresponde bien con la teoría actual en esta cuestión. De acuerdo con el ideal de sentido común, la felicidad económica consiste en el consumo ilimitado de bienes sin trabajo; por contra, el trabajo no remunerado es la perfecta aflicción económica. El hombre se rebela instintivamente contra el esfuerzo que sirve para suministrar los medios de vida.

Aunque nadie aceptaría la proposición si se expresa de esta forma tan burda, es sin embargo una exageración de algo que está implícito en los escritos de eminentes economistas. Si esta aversión al esfuerzo útil forma parte integral de la naturaleza humana, el rastro de la serpiente del Edén debería ser fácilmente reconocible por todos los hombres, porque se trata de una distinción exclusiva de la especie humana. Con seguridad, no podremos encontrar en ninguna otra especie animal una aversión similar a toda actividad dirigida al mantenimiento de la especie. Bajo el proceso selectivo a través del que se supo-

* «The Instinct of Workmanship and the Irksomeness of Labor», publicado originalmente en The American Journal of Sociology, vol. 4, 1898-1899, pp. 187-201. 
ne que las especies han aparecido y obtenido su estabilidad no hay oportunidad para la supervivencia de una especie dotada de tal aversión a la promoción de su propio proceso vital. Si el hombre es la única excepción a la norma selectiva, entonces esta extraña inclinación ha sido introducida en su carácter por algún deus ex machina malevolente.

Sin embargo, pese a todo el aparente absurdo de la cuestión, el hecho es así. Con mayor o menor sinceridad, la gente suele declarar aversión al esfuerzo útil. Esta declaración no se refiere a todo esfuerzo, sino sólo al que implica cierta utilidad; se trata, en particular, del esfuerzo vulgarmente reconocido como trabajo útil. Se expresa menor repugnancia hacia el esfuerzo que procura una ganancia pero no un producto de utilidad humana, como, por ejemplo, el esfuerzo que entraña la guerra, la política u otros empleos de naturaleza similar. Y, por lo común, no se declara aversión a los deportes u otras ocupaciones similares que no producen ni una ganancia pecuniaria ni un producto útil. Con todo, el hecho de que una determinada línea de esfuerzo sea inútil no la salva de ser odiosa, como lo demuestra el caso de los trabajos de baja categoría; muchos de estos trabajos no sirven a un fin útil, pero aun así son repugnantes para todas las personas sensibles.

El «hombre económico», cuya silueta han trazado a grandes rasgos los economistas clásicos y rellenado sus caricaturistas, es una anomalía en el mundo animal; y, sin embargo, a juzgar por las expresiones populares cotidianas, el retrato no se ha exagerado gravemente. Pero si este hombre económico debe servir de maniquí al que se debe ajustar la prenda de las doctrinas económicas, a la ciencia le corresponde explicar cuáles son sus limitaciones y cómo ha logrado emanciparse de la ley de la selección natural. Su emancipación de la ley es, de hecho, más aparente que sustancial. A este respecto, la diferencia entre el hombre y sus eventuales competidores en la lucha por la supervivencia reside en un ajuste total, no parcial y leve, de sus inclinaciones a los propósitos de la vida de la especie. Hace mucho tiempo se distanció de todos ellos en este aspecto, y la distancia es tan grande que ahora es capaz, sin riesgo para la vida de la especie, de juguetear rápida y relajadamente con la base espiritual de su supervivencia.

Al igual que otros animales, el hombre es un agente que actúa en respuesta a los estímulos que le proporciona el entorno en que vive. $\mathrm{Y}$, al igual que otras especies, es una criatura de hábitos y propensiones. Pero en un grado mayor que otras especies, el hombre asimila mentalmente el contenido de los hábitos bajo cuya dirección actúa, y aprecia la tendencia de estos hábitos y propensiones. Es, en un sentido eminente, un agente inteligente. Está dotado por necesidad selectiva de una proclividad a la acción con propósito. Posee un sentido discriminador del propósito, en virtud del cual toda futilidad de la vida o de la acción es desagradable para él. Puede haber una amplia divergencia entre los individuos con respecto a la forma y dirección en las que se expresa este impulso, pero el impulso en sí no es una cuestión de idiosincrasia, es un rasgo genérico de la naturaleza humana. No es un rasgo que se da esporádicamente en un 
puñado de individuos. Hay casos en los que esta proclividad hacia la acción intencional no existe o sólo está presente en un grado obviamente pequeño, pero las personas dotadas de este modo deficiente, propio de la acción de una madrastra, son clasificadas como «sujetos defectuosos». Las líneas de descendencia que contienen esta naturaleza humana defectuosa disminuyen y decaen incluso bajo las circunstancias más propicias de la vida moderna. La historia de las familias hereditariamente dependientes o defectuosas constituye una evidencia a estos efectos.

La gran ventaja del hombre sobre otras especies en la lucha por la supervivencia ha sido su mayor capacidad para sacar provecho de las fuerzas del entorno. Es a su proclividad a obtener ventaja de los medios materiales de vida a lo que debe su posición de señor de la creación. No es una proclividad al esfuerzo, sino al logro, a la consecución de un fin. Su primacía es, en última instancia, una primacía económica o industrial. En su vida económica el hombre es agente, no receptor; es un agente que busca en cada acto la realización de algún fin impersonal, objetivo y concreto. Como esta influyente norma de acción guía la vida de los hombres en todos los usos que hacen de las cosas materiales, debe servir también como punto de partida y proporcionar el principio orientador a toda ciencia que pretenda ser una teoría del proceso de la vida económica. En el ámbito de la teoría económica, el último análisis de cualquier fenómeno dado debe volver a este ubicuo impulso humano antes de pasar a otra cosa.

Todo esto parece contradecir lo que acabamos de decir sobre la convencional aversión al trabajo. Pero, en realidad, la contradicción no es tan grande como parece a primera vista. Su solución reside en el hecho de que la aversión al trabajo es en buena medida sólo una aversión convencional. En los momentos de reflexión sobria, cuando no está bajo la tensión del trabajo excesivo, el sentido común de los hombres habla inequívocamente bajo la guía del instinto de trabajo útil [workmanship]. A los hombres les place ver que los demás viven su vida persiguiendo algún propósito, y les place pensar que su propia vida sirve para algo. Todos los hombres tienen ese sentido cuasi estético del mérito industrial o económico, y a ese sentido del mérito económico le desagrada la ineficacia y la futilidad. En su expresión positiva es un impulso o instinto de trabajo útil; en su expresión negativa se expresa en una desaprobación del derroche. Ese sentido del mérito y el demérito con respecto al fomento o al impedimento material de la vida aprueba el acto económicamente eficaz y desaprueba la futilidad económica. No es necesario señalar en detalle la estrecha relación de esta norma del mérito económico con la norma ética de conducta, de un lado, y con la norma estética del gusto, de otro. Está relacionada de forma muy estrecha con ambas, tanto en lo que se refiere a su fundamento biológico como al alcance y al método de su recompensa.

Este instinto del trabajo útil se sitúa manifiestamente en completa contradicción con la convencional antipatía por el esfuerzo útil. Los dos se encuentran en total disonancia en el ordinario gobierno de los hombres; pero cuando quiera 
que se hace un juicio deliberado sobre conductas o acontecimientos, el primero ejerce su primacía de una forma penetrante que sugiere que en conjunto es el rasgo más genérico, más permanente, de la naturaleza humana. Apenas puede haber discusión acerca de la precedencia entre los dos. El primero es un rasgo humano necesario para la supervivencia de la especie; el segundo es un hábito de pensamiento posible sólo en las especies que han distanciado a todos sus perseguidores y prevalece entonces sólo a contracorriente y dentro de los estrictos límites que le impone el primero. Así, pues, la cuestión que se plantea respecto a ellos es: ¿es la aversión al trabajo algo derivado del instinto de trabajo útil? y ¿Cómo ha surgido y ganado solidez a pesar de no concordar con ese instinto?

Hasta hace poco tiempo ha habido entre los autores que tratan de la cultura primitiva cierto consenso en torno a que el hombre, cuando por vez primera surgió en el plano propiamente humano, tenía disposición al conflicto e inclinación a aislar sus propios intereses y propósitos de los de sus iguales y sentía predilección por las disputas y pendencias. Por consiguiente, cuando esa perspectiva se combina con la idea de que los hombres tienen una propensión natural a la acción, es evidente la presunción de que esta proclividad natural a la acción es proclividad a la acción de tipo destructivo. Se sostiene que los hombres tienden a luchar, no a trabajar, que lo normal es que el fin de la acción sea dañar más que reparar. Desde esta perspectiva, la propensión a la acción intencional se habría convertido en un impulso hacia la actividad deportiva antes que al trabajo útil. Cualquier intento de hacer encajar esta idea en un esquema de evolución de la cultura supondría la implicación de que en la fase prehumana o protoantropoide la raza era una especie predatoria y la etapa inicial de la cultura humana, así como su ulterior desarrollo cultural, fue en lo esencial de tipo predatorio.

Mucho hay que decir de esta perspectiva. Si por derivación la humanidad es una raza no de trabajadores, sino de deportistas, no hay entonces necesidad de explicar la aversión convencional al trabajo. El trabajo es no deportivo y por tanto desagradable, y la perplejidad surge entonces al explicar cómo los hombres han llegado en algún grado a reconciliarse con algo que no sea la vida predatoria. Además de la inmediata ventaja de esta perspectiva, mucha evidencia la respalda. Muchos pueblos en un estado de la cultura inferior al nuestro son de hábitos más predatorios que nosotros. La historia de la humanidad, tal y como fue convencionalmente escrita, ha sido la narración de hazañas predatorias, y por lo común no se tiene la impresión de que esta historia sea parcial o mal informada. Una inclinación deportiva a la guerra se encontrará también en casi todas las comunidades modernas. Asimismo, el sentido del supuesto honor, sea honor individual o nacional, es también una expresión de deportividad. El predominio de las nociones de honor puede, por lo tanto, tomarse como una evidencia en la misma dirección. Y, como si la pretensión de antigüedad y de reputación prescriptiva de la deportividad fortaleciera aún más, el sentido del honor es también notoriamente más intenso en las comunidades de cultura más arcaica que la nuestra. 
Ahora bien, hay un considerable cuerpo de evidencia, procedente tanto de la historia cultural como de los fenómenos actuales de la vida humana, que se opone a esa perspectiva convencionalmente aceptada que en términos genéricos hace del hombre un deportista. De forma oscura pero persistente, durante toda la historia de la cultura humana la gran masa de la gente ha estado trabajando por doquier en su vida cotidiana para que las cosas fueran de uso humano. El propósito más inmediato de todo progreso industrial ha sido el de ejecutar mejor alguna esmerada tarea. De una parte, este trabajo ha procedido necesariamente sobre la base de un interés que apreciaba la labor que había que hacer; pues no hay ninguna otra razón en que basarse para obtener algo que sea mejor que la ejecución sin propósito de una tarea. Y, de otra, la disciplina del trabajo ha actuado también necesariamente para desarrollar una actitud de destreza. Esto no equivale a decir que el trabajo realizado se deba por completo a la compulsión en régimen predatorio, pues los más notables avances a este respecto se han logrado allí donde era menor la fuerza coercitiva de la hazaña deportiva.

Las expresiones del sentido común confirman esta idea. Como ya se ha señalado, siempre que reflexionan desapasionadamente y emiten un juicio sobre el valor de la conducta humana, la tendencia común entre los hombres maduros es aprobar el instinto de trabajo útil más que la deportividad. En el mejor de los casos, adoptan una actitud de disculpa hacia esta última. Esto se puede comprobar en los actuales (mayo de 1898) disturbios producidos por la ira popular. Mientras se puede dar por sentado que la incursión bélica en que se está viendo envuelta esta comunidad es en lo esencial un arrebato de exaltación deportiva, se debe advertir que a casi todos los que hablan a favor de la guerra les cuesta encontrar un motivo aceptable de otro tipo. No se ve que la hazaña predatoria, como tal, entrañe su propia legitimación, como debería ocurrir con la percepción de la misma por cualquier especie de carácter eminentemente predador. Lo que encuentra una aprobación sin reservas es la conducta que fomenta la vida humana en su conjunto, más que la que promueve los intereses predatorios o envidiosos de uno contra otro.

Los hábitos más antiguos y más consistentes de la raza se imponen mejor cuando los hombres no hablan bajo la tensión de una irritación momentánea. Bajo estas circunstancias, la antigua disposición puede incluso destruir los apremiantes cánones de conducta convencionales. La arcaica inclinación mental que impulsa a los hombres a ensalzar la utilidad del trabajo es el resultado de una habituación larga y constante a una trayectoria vital del mismo carácter que queda reflejado en esa inclinación.

La vida del hombre es actividad; y, cuando actúa, piensa y siente. Esto ha de ser necesariamente así, puesto que es el hombre agente el que piensa y siente. Al igual que otras especies, el hombre es una criatura de hábitos y propensiones. Actúa bajo la guía de las inclinaciones que le ha impuesto el proceso de selección al que debe su diferenciación de otras especies. Es un animal social; y el proceso de selección por el que ha adquirido el armazón espiritual de un 
animal social le ha hecho ser, al mismo tiempo, un animal pacífico. La raza puede haberle alejado de la antigua posición de paz, pero incluso ahora los trazos de una tendencia pacífica en los hábitos cotidianos de pensamiento y sentimiento son muy abundantes. El espectáculo de la sangre y la presencia de la muerte, incluso de la sangre y muerte de animales inferiores, provocan por lo común en las personas inexpertas asco y repugnancia. En la mayoría de los casos, el hábito de complacencia con la matanza surge sólo como resultado de la disciplina. A este respecto, el hombre difiere de las bestias de presa. Por supuesto, difiere más ampliamente a este respecto de las bestias solitarias, pero incluso entre los animales gregarios sus parientes espirituales más cercanos no se hallan entre los carnívoros. Con su cuerpo desarmado y la escasa medida en que su fuerza muscular está especializada para la lucha, así como su aversión instintiva al contacto hostil con las bestias feroces, el hombre ha de ser clasificado antes entre los animales que deben su supervivencia a una aptitud para evitar el conflicto directo con sus competidores, que entre los que sobreviven venciendo y comiendo a sus rivales.

«El hombre es el más débil e indefenso de todos los seres vivientes»y, según la Ley de la Jungla, a él le corresponde dejarse aconsejar, idear cosas diferentes y sacar provecho de ellas en modos que son incomprensibles para los demás. Sin herramientas no es un animal peligroso, como lo son otros. Y no se convirtió en un animal formidable hasta que hizo ciertos avances en la creación de instrumentos para el combate. Antes de que las herramientas tuvieran un uso eficaz —es decir, durante la mayor parte del período de la evolución humana-, el hombre no podía ser un agente de destrucción o un perturbador de la paz. La fuerza de las circunstancias le imponía una disposición pacífica y retraída. Con el uso de las herramientas surgió gradualmente la posibilidad de que adquiriera una disposición diferente, pero incluso entonces las circunstancias que favorecían el desarrollo de una proclividad al conflicto sobrevinieron sólo de forma gradual y parcial. Los hábitos vitales de la raza aún tenían por fuerza un carácter más pacífico e industrial que destructivo y bélico. En los primeros tiempos, las herramientas y los instrumentos debieron servir sobre todo para adecuar hechos y objetos al uso humano más que para causar daño e incomodidad. La industria debió de desarrollarse mucho antes de que existiese la posibilidad de que un grupo de hombres viviera a costa de otro grupo; y durante la prolongada evolución de la industria antes de alcanzarse este punto, la disciplina de la vida en asociación se movió constantemente en la dirección de la eficacia industrial, tanto por lo que concierne a los rasgos físicos y mentales del hombre como a su actitud espiritual.

Antes de que fuese posible una vida predatoria, mediante la selección y el entrenamiento, la vida del hombre sirvió para desarrollar y conservar en él el instinto de trabajo útil. La adaptación al entorno que imponía la situación era de tipo industrial; requería que los hombres adquiriesen capacidad para adaptar cosas y situaciones al uso humano. Esto no significa sólo la adaptación de las cosas por parte del individuo a su propio uso individual, porque el hombre 
arcaico era necesariamente miembro de un grupo, y durante esta primitiva fase, cuando la eficacia industrial aún era mínima, ningún grupo podría haber sobrevivido sin la base de un sentido de la solidaridad lo suficientemente fuerte como para colocar el interés propio en un segundo plano. El interés propio, en tanto que guía aceptada de la acción, es posible sólo como elemento concomitante de una vida predatoria, y una vida predatoria es sólo posible una vez que el uso de las herramientas se ha desarrollado tanto que queda un excedente considerablemente mayor que el que se requiere para el mantenimiento de los productores. La subsistencia mediante la acción predatoria implica que exista algo sustantivo sobre lo que hacer presa.

El hombre antiguo era miembro de un grupo que para sobrevivir dependía de la eficacia industrial de sus miembros y de la unicidad de propósitos en el uso de los medios materiales de que disponía. En una fase relativamente temprana pudo darse cierta competencia entre los grupos por la posesión de los frutos de la tierra y la obtención de emplazamientos ventajosos; pero no pudo haber existido mucho contacto hostil entre los grupos, no el suficiente como para moldear los hábitos de pensamiento dominantes.

Lo que los hombres pueden hacer fácilmente es lo que hacen habitualmente, y esto decide lo que pueden pensar y saber con facilidad. Se sienten a gusto con la serie de ideas que les son familiares a su línea cotidiana de acción. Una línea habitual de acción constituye una línea habitual de pensamiento que proporciona el punto de vista desde el cual los hechos y los acontecimientos son captados y reducidos a un cuerpo de conocimiento. Lo que es coherente con la línea de acción habitual es coherente con la línea de pensamiento habitual, y proporciona la razón definitiva del conocimiento, así como el criterio convencional de satisfacción o aprobación en cualquier comunidad. Inversamente, un proceso o método de vida, una vez que se ha comprendido, asimilado por el pensamiento, opera en el esquema de vida y se convierte en una norma de conducta, simplemente porque el agente pensante, que conoce, es también el agente que actúa. Lo que es captado con facilidad y es coherente con el proceso de vida y de conocimiento es aprehendido como lo correcto y lo bueno. Todo esto se aplica con más fuerza todavía donde la habituación no es simplemente individual y esporádica, sino respaldada por el grupo o la raza por una eliminación selectiva de aquellos individuos y linajes que no se ajustan al canon exigido de pensamiento y conducta. Allí donde esto ocurre, la proclividad adquirida pasa de tener la condición de hábito a la de aptitud o propensión. Se convierte en un rasgo que puede transmitirse, y bajo su guía la acción es correcta y buena, y cuanto más duradera y más constante es la adaptación selectiva por medio de la cual surge la aptitud, más firmemente se establece esa aptitud en la raza y menos se cuestiona la sanción del canon de conducta resultante.

Por lo que se refiere a la relación del hombre con sus medios materiales de vida, el canon de pensamiento y conducta que se impuso de este modo en el hombre antiguo es lo que aquí llamamos instinto de trabajo útil. El interés que 
los hombres adquirieron por los hechos económicos sobre la base de esta propensión no tenía un carácter eminentemente egoísta antes de que la rapiña estuviera en boga. La necesaria primacía del sentido de la solidaridad del grupo lo habría impedido. El proceso de selección ha de eliminar los linajes dotados en exceso de una tendencia egoísta. Además, existía ya cierta emulación entre los individuos, incluso en los grupos más pobres y pacíficos. Debido a lo pronto que aparece un esquema de emulación allí donde las circunstancias señaladas favorecen su desarrollo, probablemente la proclividad a la emulación debió haber existido también en aquellos tiempos con la fuerza suficiente como para reafirmarse en la medida en que las exigencias de la vida antigua del grupo lo permitían. Pero esta emulación no podía ir en la dirección de una adquisición o acumulación individual de bienes, ni de una vida constantemente entregada al asalto y los tumultos. Debió de ser una emulación como la que se encuentra entre los animales gregarios pacíficos; es decir, era primaria y principalmente emulación sexual, recurrente con mayor o menor regularidad. Más allá de esto, debió de haber existido cierta disputa por la distribución de los bienes disponibles, pero ni ésta ni la rivalidad por las subsistencias podrían haber sido el tono dominante de la vida.

Bajo el canon de conducta impuesto por el instinto de trabajo útil, la eficacia, la utilidad, es alabada por sí misma, y la ineficacia y la futilidad resultan odiosas. El hombre contempla su propia conducta y la de sus vecinos y emite un juicio de complacencia o de desprecio. El grado de eficiencia con que se adapta a los criterios aceptados de eficacia determina en gran medida su satisfacción consigo mismo y con su situación. Una discrepancia amplia o persistente a este respecto es fuente de gran malestar espiritual.

De este modo puede hacerse un juicio sobre la intención del agente o sobre la utilidad del acto. En el primer caso, la atribución de mérito o demérito se cataloga como moral; pero la atribución de méritos de este tipo no se tratará en este trabajo. En lo que se refiere a la utilidad o a la eficacia, los hombres no sólo adquieren un pensamiento de primera mano sobre los hechos de su propia conducta, sino que son sensibles a la reprimenda o la aprobación de los otros. No es sólo que la conciencia inmediata del logro de un propósito sea gratificante y estimulante, sino que la imputación de eficacia por parte de un igual tal vez sea no menos gratificante y estimulante.

La sensibilidad a la reprimenda o a la aprobación es cuestión de necesidad selectiva bajo las circunstancias de la vida en asociación. Sin ella, ningún grupo de hombres podría sobrellevar una vida colectiva en un entorno material que exige adaptar los fines del hombre. En este aspecto el hombre muestra de nuevo una relación espiritual con los animales gregarios antes que con las bestias solitarias de presa.

Bajo la guía de este gusto por el buen trabajo, los hombres se comparan unos con otros y con los ideales aceptados de eficacia, y son valorados y clasificados por el sentido común de sus iguales de acuerdo con un esquema convencional de mérito y demérito. La imputación de eficacia necesariamente sigue a 
la evidencia de eficacia. El logro visible de un hombre es, por lo tanto, comparado con el de otro, y la concesión de estima se basa habitualmente en una comparación envidiosa entre las personas en lugar de en el ajuste inmediato a la línea de conducta adecuada para alcanzar el fin aprobado de la acción. La causa de la estima así atribuida deja de ser una evaluación directa de la conveniencia de una conducta para convertirse en una comparación entre las capacidades de los diferentes agentes. En lugar de una valoración de la utilidad, se calibra la capacidad sobre la base del éxito visible. Y lo que llega a compararse en una comparación envidiosa de este tipo entre los agentes es la fuerza que el agente es capaz de aplicar, más que la utilidad de su conducta. Por consiguiente, en tanto en cuanto la estima concedida a la utilidad se convierte en una estima envidiosa de un agente comparado con otro, el fin que persigue la acción dejará de ser la simple conveniencia para convertirse en una manifestación de capacidad o fuerza. Poner de manifiesto la evidencia del poder, en lugar de conseguir un fin impersonal por sí mismo simplemente como artículo de uso humano, se convierte en el fin inmediato del esfuerzo. De manera que, mientras en su expresión más inmediata la norma del gusto económico se mantiene como un impulso hacia la laboriosidad o un gusto por la utilidad y como un disgusto por la futilidad, en determinadas circunstancias de la vida en asociación llega en alguna medida a adquirir el carácter de una demostración emuladora de fuerza.

Puesto que la imputación de eficacia y de mérito envidioso depende de la evidencia que proporciona el éxito visible, debe evitarse la apariencia del mal si se quiere eludir el desprecio. En la antigua cultura salvaje, mientras el grupo es pequeño y todavía no se dan las condiciones favorables a la vida predatoria, la emulación resultante entre los miembros del grupo favorece especialmente la eficacia industrial. Lo que llega a ser evitado es la apariencia de incapacidad industrial. Es en esta dirección en la que la fuerza o capacidad se pone de manifiesto con la mayor consistencia y los mejores resultados para el buen nombre del individuo. Por tanto, es en esta dirección en la que se desarrollará un criterio del mérito y un canon de la conducta meritoria. Pero ni siquiera para el desarrollo progresivo de la emulación en el uso productivo del cerebro y los músculos es el grupo pequeño, rudo y pacífico de salvajes terreno abonado. La situación no favorece un espíritu de emulación vigoroso. Las condiciones favorables para el desarrollo de un hábito de demostración emuladora de fuerza son: 1) la repetición frecuente de coyunturas que requieren un esfuerzo grande y súbito, y 2) la exposición del individuo a un entorno humano grande y en especial cambiante, en el que busca su aceptación. Estas condiciones no se cumplen de modo eficaz en los niveles más bajos de salvajismo, tal y como la cultura humana debió de haber sido en los primeros tiempos del uso de las herramientas. Por consiguiente, se observa relativamente poco espíritu emulador en las comunidades que han conservado la antigua constitución pacífica o que han regresado a ella desde una cultura superior. En estas comunidades se da un nivel bajo de cultura y confort, junto a una falta de aplicación tenaz al 
trabajo y a una relativa ausencia de envidias y gradaciones entre rangos. Las nociones de rango económico y discriminación entre las personas, por lo que concierne bien a las posesiones, bien al confort, están siempre o casi siempre latentes.

Con el desarrollo posterior del uso de las herramientas y del control humano de las fuerzas del entorno cambian los hábitos de vida del grupo salvaje. Es probable que haya más agresión, tanto en la práctica de la caza mayor como en los conflictos entre grupos. A medida que aumenta la eficacia industrial del grupo y las armas alcanzan mayor perfección, aumentan también los incentivos para la agresión y las oportunidades de logro que se derivan de utilizarla. Se cumplen plenamente, pues, las condiciones favorables para la emulación. Con el aumento de la densidad de la población que se sigue de una mayor eficacia industrial, el grupo pasa, forzado por las circunstancias, desde una condición antigua de paz azotada por la pobreza a una fase de vida predatoria. La fase combativa —el comienzo de la barbarie- puede implicar la acción predadora agresiva o también que el grupo se coloque simplemente a la defensiva. Una u otra, o ambas líneas de actividad - y, sin duda, por lo general ambas-, se impondrán en el grupo, bajo pena de exterminio. Éste fue, por lo visto, el curso normal de la temprana evolución social.

Cuando un grupo entra en esta fase predadora de su desarrollo, los empleos que más ocupan la atención de los hombres son los empleos que implican hazaña. La preocupación más importante del grupo, y al mismo tiempo la dirección en la que el individuo puede alcanzar un efecto más espectacular, es el conflicto con los hombres y las bestias. Resulta fácil hacer una reveladora comparación entre los hombres cuando su trabajo consiste en una serie de hazañas realizadas contra esos difíciles adversarios o contra los formidables movimientos de los elementos. La demostración de una mano fuerte, de una agresión triunfante, usualmente de carácter destructivo, se convierte en el fundamento aceptado de la reputación. El interés vital dominante del grupo arroja su fuerte luz sobre este encomiable empleo de la fuerza y la sagacidad, y las otras, más oscuras, formas de servir a la vida del grupo pasan a un segundo plano. El ánimo que guía al grupo se torna militar, y las acciones de los hombres son juzgadas desde el punto de vista del hombre que lucha. Lo que sin crítica ni recelo se reconoce como útil y eficaz en tales grupos es la capacidad de lucha. La hazaña se convierte en la base convencional de la comparación envidiosa entre individuos y la reputación termina por descansar en el valor.

A medida que la cultura predatoria alcanza un desarrollo más completo, surge una distinción entre empleos. La tradición del valor, como virtud par excellence, gana en alcance y robustez hasta casi llegar a ser reconocida como la virtud única. Así, pues, sólo son valiosos y renombrados los empleos que implican el ejercicio de esa virtud. Otros empleos, en los que los hombres se ocupan adaptando suavemente los materiales inertes al uso humano, se vuelven indignos y terminan por ser degradantes. El hombre honorable debe no sólo mostrar capacidad para la proeza predatoria, sino también evitar enredarse en ocupaciones que no entrañan hazaña. Los empleos tranquilos que no implican una destruc- 
ción obvia de la vida ni una coerción espectacular de los rivales recalcitrantes caen en el descrédito y se relegan a aquellos miembros de la comunidad con algún defecto en su capacidad de predación; es decir, a aquellos que carecen de grandeza, agilidad o ferocidad. La ocupación en estos empleos supone que la persona que los realiza carece de ese mínimo aceptado de valor que le daría derecho a ser considerado como un hombre de buena posición. Para tener una reputación inmaculada se debe evitar la apariencia del mal. Por lo tanto, el bárbaro sano de la cultura predatoria, que es por completo consciente de su buen nombre, deja estrictamente todos los trabajos pesados y monótonos a las mujeres y los menores del grupo. Dedica su tiempo al arte varonil de la guerra y su talento a idear modos y medios de perturbar la paz. Sobre esta base descansa el honor.

En el esquema bárbaro de vida los empleos industriales y pacíficos corresponden a las mujeres. Requieren poca fuerza, incapacidad para la agresión y devastación y, por tanto, carecen de buena reputación. Pero todo lo que se acepta como señal convencional de defecto o vicio llega pronto a explicarse como intrínsecamente bajo. Así, las ocupaciones industriales provocan un odio educado y se perciben como sustancialmente innobles. Son no deportivas. El trabajo mancha, y los hombres que se respetan a sí mismos deben evitar toda contaminación con empleos vulgares.

Allí donde la cultura predatoria se ha desarrollado plenamente, la percepción de sentido común de que el trabajo es innoble ha evolucionado para convertirse en el concepto más refinado de que el trabajo es malo para los que aún no han caído en el descrédito. De aquí la razón de ser de ciertos rasgos bien conocidos de la casta y el tabú. En el desarrollo cultural posterior, una vez que se ha acumulado cierta riqueza y los miembros de la comunidad se dividen entre, de un lado, una clase servil y, de otro, una clase ociosa, la tradición de que el trabajo es innoble adquiere una importancia adicional. No es sólo un indicio de fuerza inferior, sino también un gaje del pobre. Ésta es la situación de nuestros días. El trabajo es moralmente imposible debido a la antigua tradición que nos ha llegado desde la temprana barbarie, y es vergonzoso debido a su funesta asociación con la pobreza. Es indecoroso.

El fastidio del trabajo es un hecho espiritual; reside en su indignidad. Con todo, el hecho del fastidio no resulta menos real ni menos eficaz porque su carácter sea de tipo espiritual. De hecho, es lo más sustancial e irremediable en esta explicación. La molestia física y su carácter desagradable se pueden soportar sólo si está presente el incentivo espiritual. Lo confirma el atractivo de la guerra tanto para el joven bárbaro como para el civilizado. Los relatos más comunes de la experiencia de un guerrero incluyen dramáticas sugerencias de privación, exposición a las inclemencias del tiempo, fatiga, alimañas, miseria, enfermedad y muerte repugnante; al margen del poder de las palabras, los incidentes y accesorios de la guerra se consideran asquerosos, feos y malsanos. La guerra es una ocupación atractiva sólo si uno está dotado del apropiado hábito mental. La mayoría de los deportes y muchos otros empleos cultos que son dolorosos pero dignos de elogio son evidencia del mismo efecto. 
El fastidio físico es una incomodidad que los hombres no se suelen tomar en serio si no va reforzado por la sanción del decoro; pero otra cosa distinta sucede con el fastidio espiritual de tal trabajo en tanto es condenado por el uso educado. Esto es un hecho cultural. No hay remedio para este tipo de fastidio, a no ser una subversión de la estructura cultural sobre la que descansan nuestros cánones de decencia. Por supuesto, podríamos apelar al gusto y la conciencia para anular la aversión convencional al trabajo; de vez en cuando las personas bienintencionadas y optimistas hacen una tal apelación, y de este modo se han obtenido algunos resultados intermitentes. Pero, en esto, el hombre corriente, que actúa con sentido común, está obligado a cumplir los mandatos del decoro que se derivan del sentido común: la herencia de un linaje cultural ininterrumpido que regresa al principio.

(Traducción de M. ${ }^{a}$ Teresa CASAdO.) 